\title{
Evolution of a domain wall in expanding universe: inflation and after it
}

\author{
A.D. Dolgov ${ }^{1,2, *}$, S.I. Godunov ${ }^{1,2, * *}$, and A.S. Rudenko ${ }^{1,3, * * *}$ \\ ${ }^{1}$ Physics Department and Laboratory of Cosmology and Elementary Particle Physics, \\ Novosibirsk State University, Pirogova st. 2, Novosibirsk, 630090 Russia \\ ${ }^{2}$ Theory Division, Institute for Theoretical and Experimental Physics, \\ Bolshaya Cheremushkinskaya st. 25, Moscow, 117218 Russia \\ ${ }^{3}$ Theory Division, Budker Institute of Nuclear Physics, \\ akademika Lavrentieva prospect 11, Novosibirsk, 630090 Russia
}

\begin{abstract}
We study the evolution of thick domain walls in the expanding universe. We have found that the domain wall evolution crucially depends on the time-dependent parameter $C(t)=1 /\left(H(t) \delta_{0}\right)^{2}$, where $H(t)$ is the Hubble parameter and $\delta_{0}$ is the width of the wall in flat space-time. For $C(t)>2$ the physical width of the wall, $a(t) \delta(t)$, tends with time to constant value $\delta_{0}$, which is microscopically small. Otherwise, when $C(t) \leq 2$, the wall steadily expands and can grow up to a cosmologically large size.
\end{abstract}

\section{Introduction}

Creation of the baryon asymmetry of the universe and possible existence of the cosmological antimatter crucially depends upon the version of $C$ and $C P$ violation realized in the early universe. In this connection spontaneous $C P$ violation suggested in paper [1] is of particular interest. This beautiful model, however, suffers from the domain wall problem [2]. To avoid this problem the mechanism of the wall destruction was proposed, see e.g. [3] and references therein. Another problem of the baryogenesis based on spontaneous $C P$ violation is the width of the wall. In flat space-time the width of the wall is microscopically small and if the walls with such or similar widths were created in the cosmological situation, the matterantimatter domains would be in close contact with each other. It would lead to very large annihilation rate and to unacceptably high background of the annihilation products in the universe. However, the cosmological expansion may lead to much larger separation of the domains eliminating or smoothing down this problem.

The evolution of the domain wall width in de Sitter universe was studied in the papers [4, 5]. In what follows we study the wall evolution in the universe with the equation of state $p=w \rho$ and in the inflationary universe. We show that there exists some range of the inflation parameters leading to a large domain separation prior to baryogenesis. This talk is based on papers $[5,6]$.

\footnotetext{
*e-mail: dolgov@fe.infn.it

**e-mail: sgodunov@itep.ru

***e-mail: a.s.rudenko@inp.nsk.su
} 


\section{Evolution of thick domain walls in de Sitter universe}

We consider a model of real scalar field $\varphi$ with a simple double-well potential. The Lagrangian of such model is the following,

$$
\mathcal{L}=\frac{1}{2} g^{\mu \nu} \partial_{\mu} \varphi \partial_{\nu} \varphi-\frac{\lambda}{2}\left(\varphi^{2}-\eta^{2}\right)^{2}
$$

The equation of motion of field $\varphi$ can be easily obtained from (1) and looks as

$$
\frac{1}{\sqrt{-g}} \partial_{\mu}\left(\sqrt{-g} g^{\mu v} \partial_{\nu} \varphi\right)=-2 \lambda \varphi\left(\varphi^{2}-\eta^{2}\right)
$$

In flat space-time with the metric, $d s^{2}=d t^{2}-\left(d x^{2}+d y^{2}+d z^{2}\right)$, and in static onedimensional case, $\varphi=\varphi(z)$, the equation (2) has a kink-type solution, describing a static infinite flat domain wall in $x y$-plane. Without loss of generality we can assume that the wall is situated at $z=0, \varphi(z)=\eta \tanh \frac{z}{\delta_{0}}$, where $\delta_{0}=1 /(\sqrt{\lambda} \eta)$ is the width of the wall in flat space-time.

The evolution of thick domain walls in spatially flat section of the de Sitter universe with the metric $d s^{2}=d t^{2}-e^{2 H t}\left(d x^{2}+d y^{2}+d z^{2}\right)$, with a constant Hubble parameter, $H>0$, was considered previously in papers $[4,5]$. All parameters are combined into a single positive constant, $C=1 /\left(H \delta_{0}\right)^{2}=\lambda \eta^{2} / H^{2}>0$, so it is quite natural that the evolution of domain walls is determined only by the value of $C$. In the case of very thin domain walls, $\delta \ll H^{-1}$, i.e. $C \gg 1$, the solution $\varphi(z)$ is well approximated by the flat-spacetime solution. For sufficiently large value of parameter $C, C>2$, the initial kink configuration in a de Sitter background tends to the stationary solution which depends only on physical distance $l=a(t) z$, i.e. $\varphi=$ $\eta f(H l)=\eta f\left(e^{H t} H z\right)$. The width of the stationary wall rises with decreasing value of $C$.

Above the critical value, $\delta_{0} \geq H^{-1} / \sqrt{2}$, i.e. $C \leq 2$, there are no stationary solutions at all. But if one allows for an arbitrary dependence of the solution on $z$ and $t$, the solution exists for any $C$, and the case of $C \leq 2$ leads to the expanding kink with rising width.

\section{Evolution of thick domain walls in $p=w \rho$ universe}

In an expanding universe with the equation of state of matter $p=w \rho$, where constant $w>-1$, the scale factor increases as some power of time,

$$
a(t)=\text { const } \cdot t^{\alpha}, \quad \text { where } \alpha=\frac{2}{3(1+w)}>0, \text { and the Hubble parameter } H(t)=\frac{\dot{a}}{a}=\frac{\alpha}{t} .
$$

The values $w=0(\alpha=2 / 3)$ and $w=1 / 3(\alpha=1 / 2)$ correspond to the matter-dominated and radiation-dominated universe, respectively.

The equation of motion (2) in the case when the field is a function of only $z$ and $t$ :

$$
\frac{\partial^{2} f}{\partial t^{2}}+3 H(t) \frac{\partial f}{\partial t}-\frac{1}{a^{2}(t)} \frac{\partial^{2} f}{\partial z^{2}}=\frac{2}{\delta_{0}^{2}} f\left(1-f^{2}\right),
$$

where $f(z, t)=\varphi(z, t) / \eta$. Due to the feature of the $p=w \rho$ universe (see details in [6]) one can eliminate the explicit dependence on $\delta_{0}$ in (4). This means that the equation of motion is the same for different $\delta_{0}$ if $t$ and $z$ are measured in units of $\delta_{0}$. Till the end of this section we will use $(t, z)$ notations considering $\delta_{0}=1$.

The boundary conditions for the kink-type solution should be

$$
f(0, t)=0, \quad f( \pm \infty, t)= \pm 1,
$$


and we choose the following initial configuration:

$$
f\left(z, t_{i}\right)=\tanh \frac{z \cdot a\left(t_{i}\right)}{\delta_{0}},\left.\quad \frac{\partial f(z, t)}{\partial t}\right|_{t=t_{i}}=0 .
$$

For the scale factor we choose the following form: $a(t)=a_{0} \cdot\left(t / t_{i}\right)^{\alpha}$ with the constant $a_{0}=1$, so $a\left(t_{i}\right)=1$ which means that the initial coordinate and physical distances are the same.

The results of numerical calculation for the time dependence of physical width of the wall, $a(t) \delta(t)$, in radiation-dominated and matter-dominated universe are presented in Fig. 1 for $t_{i} / \delta_{0}=0.5$ and $t_{i} / \delta_{0}=1.0$ along with plots for other values of $w$. Here $\delta(t)$ is the coordinate thickness of the wall (details in [6]).

The evolution of the domain wall is basically defined by the parameter $C(t)$. In the $p=w \rho$ universe the parameter $C(t)$ increases as $C(t)=t^{2} /\left(\alpha \delta_{0}\right)^{2} \propto t^{2}$. Since $C=2$ is the critical value, let us introduce the time $t_{C}$ at which $C\left(t_{C}\right)=2$. In $p=w \rho$ universe

$$
\frac{t_{C}}{\delta_{0}}=\sqrt{2} \alpha \Rightarrow t_{C}>t_{i} \text { for } w<\frac{2 \sqrt{2}}{3} \frac{\delta_{0}}{t_{i}}-1 .
$$

For $t_{i} / \delta_{0}=1.0$ we get that $t_{C}>t_{i}$ for $w<\frac{2 \sqrt{2}}{3}-1 \approx-0.057$. In this case $C(t)>2$ during all the time of the wall evolution for $w=1 / 3$ and $w=0$, while for $w=-1 / 3$ $(\alpha=1, \dot{a}(t)=$ const $), w=-2 / 3(\alpha=2, \ddot{a}(t)=$ const $), w=-7 / 9(\alpha=3, \dddot{a}(t)=$ const $)$ at the initial moment $C<2$. In Fig. 1 one can see that in this case while $C<2$ the physical width of the wall, $a(t) \delta(t)$, increases rapidly.

In Fig. 1 we presented the domain walls with the initial physical width of the ones in stationary universe $(H=0)$. By doing that, we are separating the influence of cosmological expansion from the natural shrinking/expanding of the wall when it approaches stationary solution. However, we can consider the evolution of the wall width from different initial configurations. In Fig. 2 we can see the evolution of the wall width from the configurations stretched along $z$ by factor $k$ :

$$
f\left(z, t_{i}\right)=\tanh \frac{z \cdot a\left(t_{i}\right)}{k \cdot \delta_{0}} .
$$

For $w=1 / 3$ (so $t_{C}<t_{i}=1 \cdot \delta_{0}$ ) all initial configurations quickly approach their common stationary solution. On the other hand, for $w=-2 / 3$ (so $t_{C}>t_{i}=1 \cdot \delta_{0}$ ) there is a period of growth for all initial configurations though it is worth noting that this growth depends on $k$.

To conclude this section, we note that in $p=w \rho$ universe it is difficult to obtain domain walls with cosmologically large width ( $w$ should be really close to -1 for that). Such domain walls can exist at the beginning of $p=w \rho$ stage. However, in such universe the parameter $C(t)$ increases, so at some moment the wall width starts to shrink and eventually goes to the constant value, $\delta_{0}$, which is microscopically small.

\section{Evolution of thick domain walls in inflationary universe}

Let us consider a simple model of inflation with quadratic inflaton potential $U=m^{2} \Phi^{2} / 2$. Although the model of inflation with a quadratic potential is really constrained in the view of the recent observational data, only the very fact of exponential expansion is important here, regardless of the specific mechanism of inflation.

The Hubble parameter and the scale factor in the slow-roll regime have the form:

$$
a(t)=a_{0} \cdot \exp \left(\sqrt{\frac{4 \pi}{3}} \frac{m}{m_{p l}} \Phi_{i} t-\frac{1}{6} m^{2} t^{2}\right) \text { and } H(t)=\sqrt{\frac{4 \pi}{3}} \frac{m}{m_{p l}} \Phi_{i}-\frac{1}{3} m^{2} t,
$$




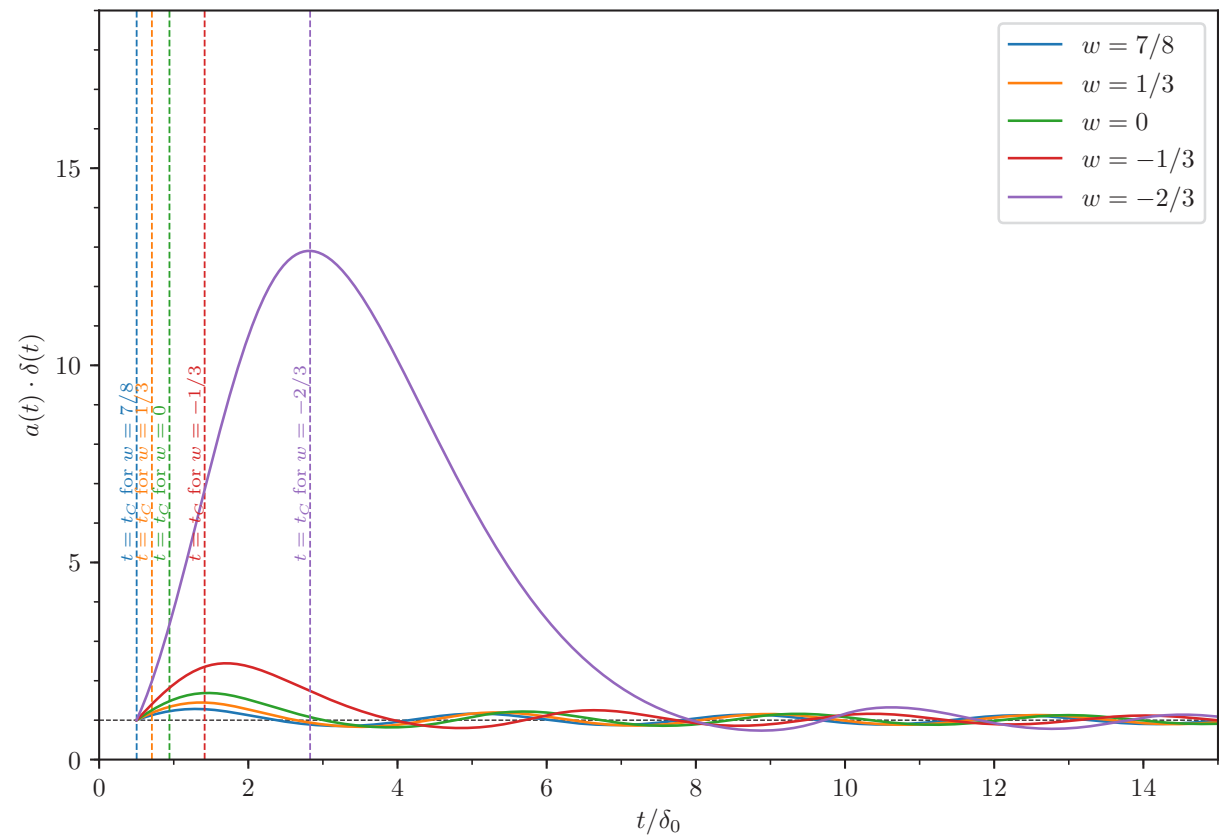

(a) $t_{i} / \delta_{0}=0.5$

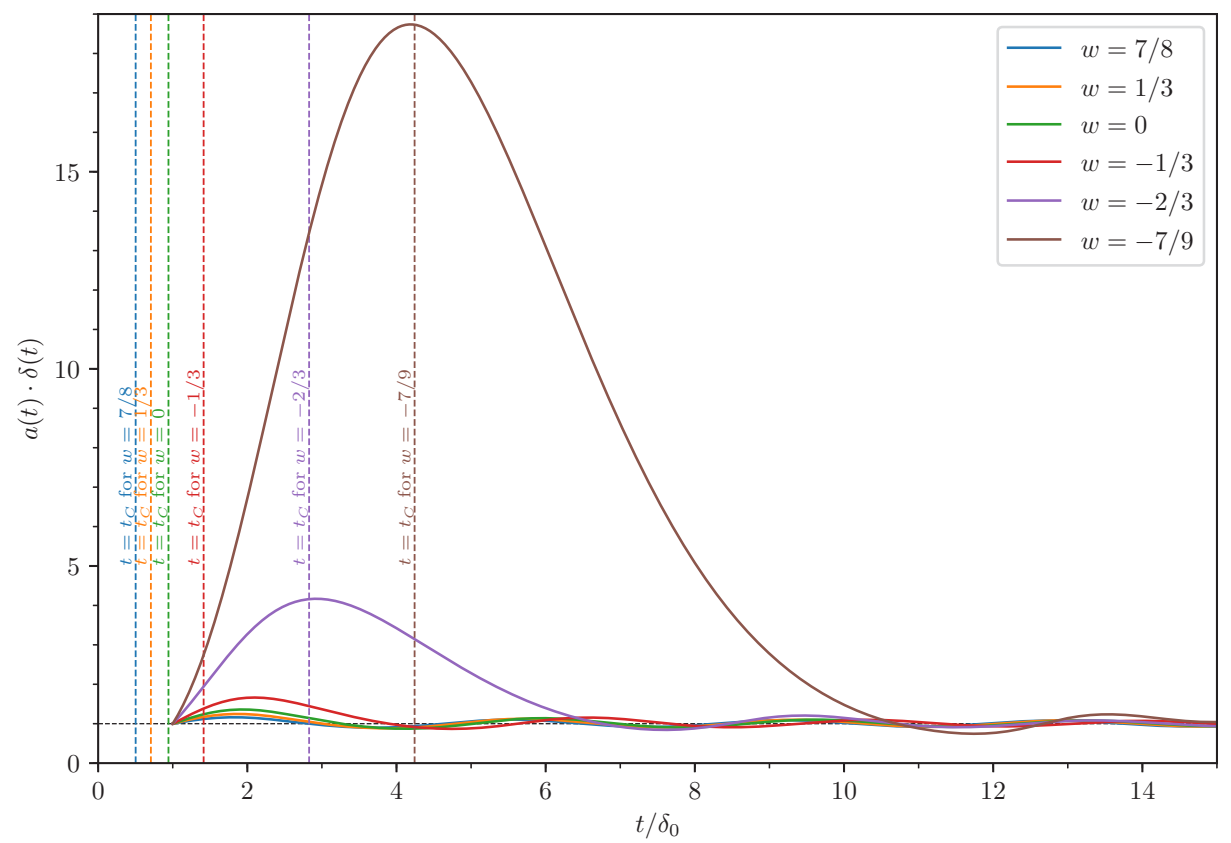

(b) $t_{i} / \delta_{0}=1.0$

Figure 1: Time dependence of the physical width of the wall, $a(t) \delta(t)$, for different values of parameter $w$. Dashed horizontal line corresponds to $\delta_{0}$. Vertical dashed lines correspond to the moment $t_{C}$ at which $C\left(t_{C}\right)=2$ (the correspondence between color and $w$ is the same). 


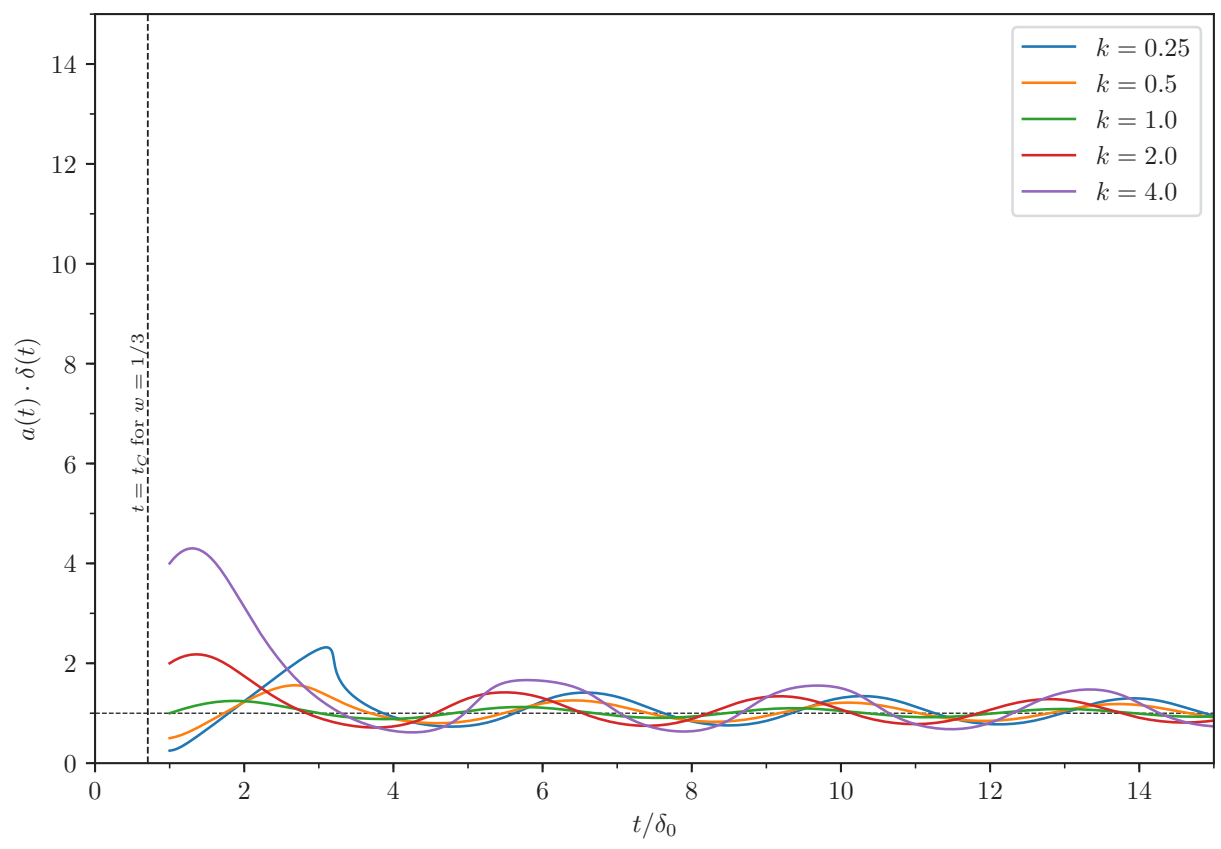

(a) $w=\frac{1}{3}$

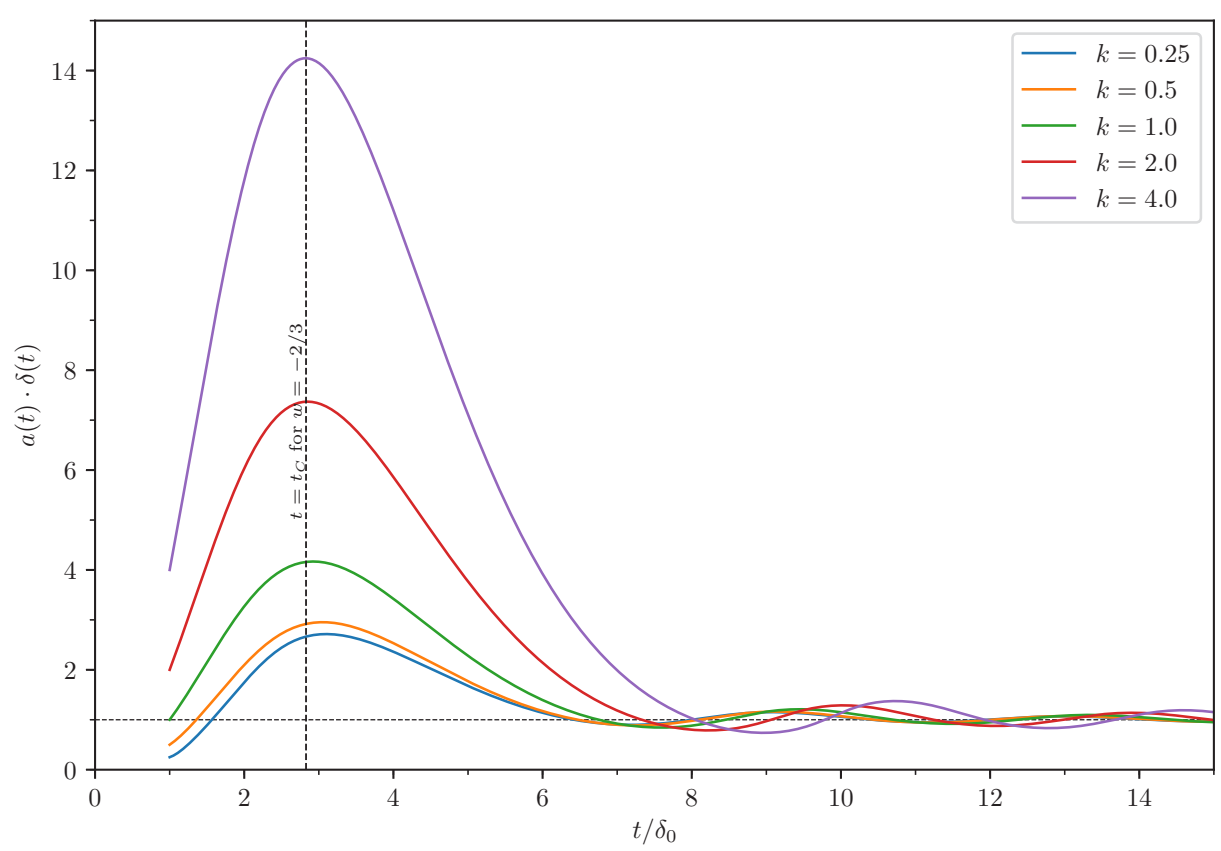

(b) $w=-\frac{2}{3}$

Figure 2: Time dependence of the physical width of the wall, $a(t) \delta(t)$, for different values of stretch parameter $k$. Dashed horizontal line corresponds to $\delta_{0}$. For both plots $t_{i} / \delta_{0}=1.0$. Vertical dashed lines correspond to the moment $t_{C}$ at which $C\left(t_{C}\right)=2$. 
where $m_{p l}$ is the Planck mass, $m$ is the inflaton mass. These formulas are valid only till the end of inflation, i.e. till the violation of slow-roll conditions. However, we use them a little longer, till $t_{e} \equiv \sqrt{12 \pi}\left(\Phi_{i} / m_{p l}\right) m^{-1}$, for which $\Phi\left(t_{e}\right)=0, H\left(t_{e}\right)=0$.

It is convenient to use $1 / m$ units in equation of motion (4):

$$
\frac{\partial^{2} f}{\partial(t \cdot m)^{2}}+m\left(t_{e}-t\right) \frac{\partial f}{\partial(t \cdot m)}-\frac{1}{a^{2}(t)} \frac{\partial^{2} f}{\partial(z \cdot m)^{2}}=\frac{2}{\left(m \cdot \delta_{0}\right)^{2}} f\left(1-f^{2}\right) .
$$

The boundary conditions should be the same as in (5). In numerical calculations we choose initial configuration given by (6) and use the following values: $\Phi_{i}=2 m_{p l}, t_{i}=0$, and $a_{0}=1$.

Time $t_{C}$ at which $C\left(t_{C}\right)=2$ is determined from the relation $m \cdot t_{C}=m t_{e}-\frac{3 \sqrt{2}}{2 m \delta_{0}}$. Parameter $C(t)$ can be equal 2 only if $t_{C} \geq 0$, i.e. $m \delta_{0} \geq 3 \sqrt{2} /\left(2 m t_{e}\right)=\sqrt{3} m_{p l} /\left(2 \sqrt{2 \pi} \Phi_{i}\right) \approx 0.173$.

Time dependence of physical width of the wall, $a(t) \delta(t)$, for different values of initial wall width, $\delta_{0}$, is shown in Fig. 3. When $\delta_{0}$ is so small that $C(t)>2$ during all the time of inflation, then the domain wall width $a(t) \delta(t)$ after some damped oscillations tends to constant value, $\delta_{0}$, which is microscopically small. For larger $\delta_{0}$, such that $C(t)$ became larger than 2 only near the end of inflation, domain wall width could grow to cosmologically large size before that moment (see Figs. 3g-3h).

\section{Conclusions}

We have found that at inflationary epoch the width of the domain walls exponentially rises when the parameter $C(t)$ is smaller than the critical value $C=2$. Therefore, the wall width might rise up to cosmologically large scales if $C(t)$ remained smaller than 2 till the end of inflation. However, when inflation is over and the expansion turns into the power law regime with $H$ decreasing with time as $1 / t$, the parameter $C \sim 1 / H^{2}$ rises above $C=2$ at some stage, and the wall started to shrink.

Our original scenario [3] spans throughout the inflation and ends in the beginning of the reheating stage where the baryogenesis is supposed to take place. That is why we are interested in the wall evolution during all the inflation and in the beginning of the $p=w \rho$ stage. The main result of our calculations is that there exists sufficiently wide range of the parameters for which the domain wall remains astronomically wide at the baryogenesis epoch. This ensures large separations between domains of matter and antimatter and prevents from catastrophic annihilation.

\section{Acknowledgements}

SG thanks the Organizers for the opportunity to give this talk. SG is also grateful to T. Prokopec and A. Starobinsky for the interest in the subject of this talk and great questions.

Authors acknowledge support of the RSF Grant No. 16-12-10037. SG is also supported under the RFBR grants No. 16-32-60115, 16-02-00342. 


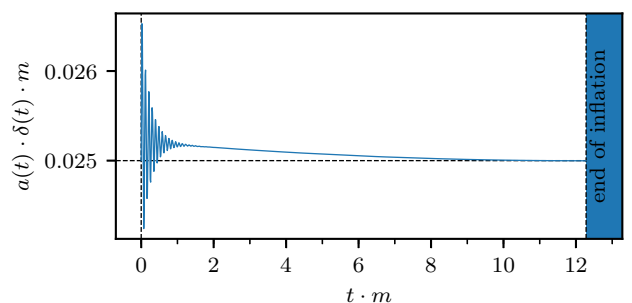

(a) $\delta_{0}=0.025 \cdot m^{-1}$

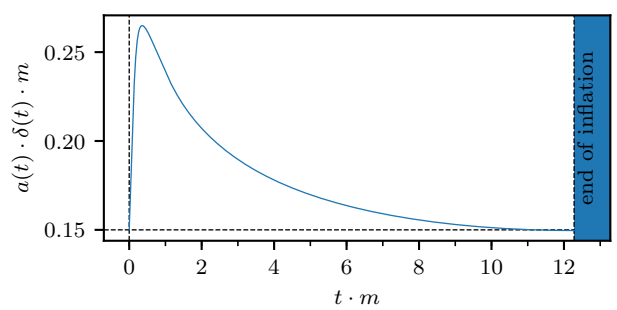

(c) $\delta_{0}=0.15 \cdot m^{-1}$

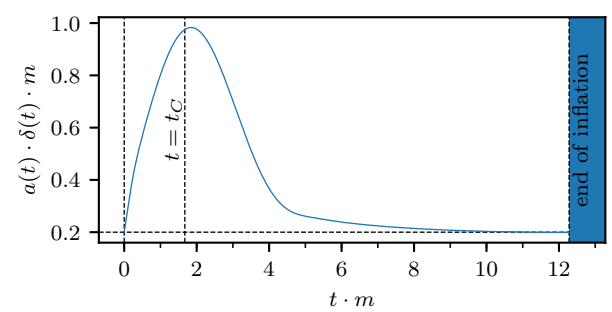

(e) $\delta_{0}=0.2 \cdot m^{-1}$

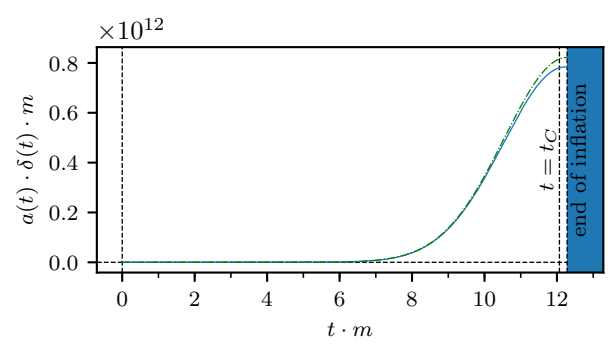

(g) $\delta_{0}=10 \cdot m^{-1}$

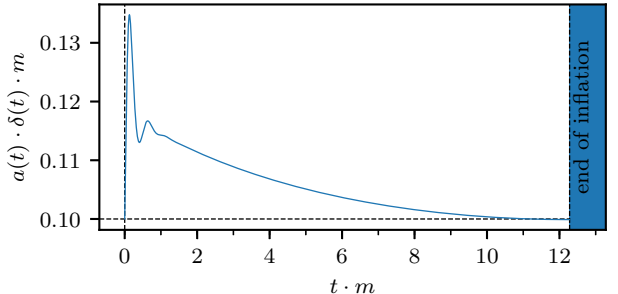

(b) $\delta_{0}=0.1 \cdot m^{-1}$

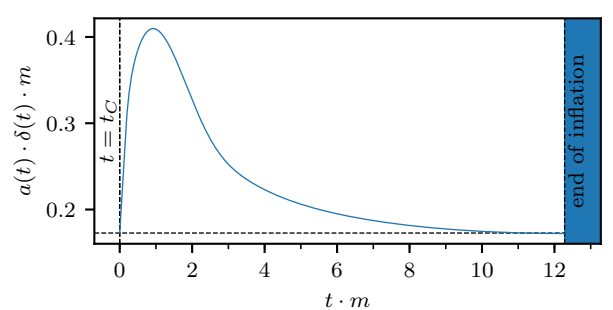

(d) $\delta_{0} \approx 0.173 \cdot m^{-1}$

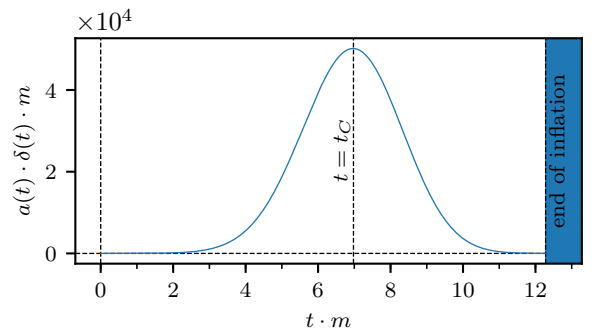

(f) $\delta_{0}=0.4 \cdot m^{-1}$

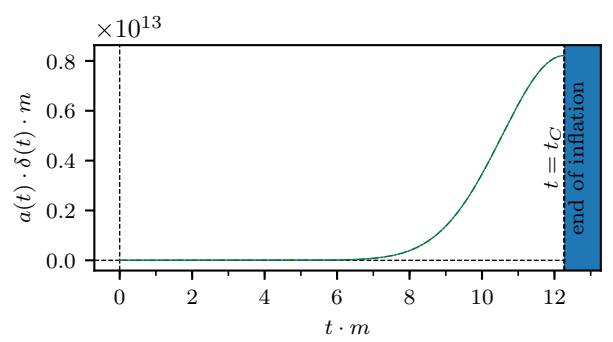

(h) $\delta_{0}=100 \cdot m^{-1}$

Figure 3: Time dependence of physical width of the wall, $a(t) \cdot \delta(t) \cdot m$, for different values of initial wall width, $\delta_{0}$. Dashed horizontal line corresponds to $\delta_{0}$. Time $t_{e}$ is shown by vertical dashed line with the corresponding label. In Figs. 3d-3h there is a vertical dashed line which corresponds to $t=t_{C}$. In Figs. $3 \mathrm{~g}$ and $3 \mathrm{~h}$ there is a green (dash-dotted) line which corresponds to $a(t) \cdot \delta_{0} \cdot m$. In Fig. 3h this line almost coincides with $a(t) \cdot \delta(t) \cdot m$. 


\section{References}

[1] T.D. Lee, Phys. Rev. D8, 1226 (1973)

[2] Ya.B. Zeldovich, I.Yu. Kobzarev, L.B. Okun, Zh. Eksp. Teor. Fiz. 67, 3 (1974), [Sov. Phys. JETP 40, 1 (1974)]

[3] A.D. Dolgov, S.I. Godunov, A.S. Rudenko, I.I. Tkachev, JCAP 1510, 027 (2015), arXiv: 1506.08671

[4] R. Basu, A. Vilenkin, Phys. Rev. D50, 7150 (1994), gr-qc/9402040

[5] A.D. Dolgov, S.I. Godunov, A.S. Rudenko, JCAP 1610, 026 (2016), arXiv: 1609.04247

[6] A.D. Dolgov, S.I. Godunov, A.S. Rudenko (2017), arXiv: 1711.04704 